\title{
Concepciones sobre Educación Inclusiva y su relación con la práctica pedagógica de los docentes: un estudio de caso
}

\section{Conceptions about Inclusive Education and its relationship with the pedagogical practice of teachers: A case study}

\author{
* Nilsa Shirley Benítez \& ** Paty Pacheco Herrera
}

Benítez, N. S., \& Pacheco, P. (2021). Concepciones sobre Educación Inclusiva y su relación con la práctica pedagógica de los docentes: un estudio de caso. Revista Convergencia Educativa, (9), julio, 16-29.

DOI: http://doi.org/10.29035/rce.9.16

[Recibido: 19 diciembre, 2020 / Aceptado: 08 junio, 2021]

\section{RESUMEN}

La Educación Inclusiva (EI) se centra en la atención a la diversidad, en la medida en que, a través de procesos administrativos y pedagógicos, busca reconocer y potencializar las fortalezas de todos los estudiantes en situación de vulnerabilidad, atendiendo a sus diferentes estilos, ritmos y particularidades en el aprendizaje. Desde esta perspectiva, se planteó la presente investigación desarrollada en una Institución Educativa de la ciudad de Montería-Córdoba, la cual estuvo encaminada a determinar las relaciones que existen entre las concepciones que tienen los docentes sobre la Educación Inclusiva (E.I) y las relaciones que existen entre estas con la práctica pedagógica que desarrollan en sus aulas. Para alcanzar este cometido, la metodología implementada obedeció al enfoque cualitativo, con diseño fenomenológico, lo cual permitió evidenciar las experiencias de los docentes y rescatar dichas voces inmersas que dan cuenta si existe o no relación entre lo que dicen y hacen en cuanto a la implementación de la E.I. Para recolectar la información se utilizaron tres técnicas: una lectura de contexto, una entrevista semi-estructurada a ocho docentes de educación básica secundaria de los grados $6^{\circ}$ a $11^{\circ}$ y se examinaron tres sesiones de clases diferentes por cada profesor, registrando las referencias a través de un protocolo de observación. A partir del análisis de los datos percibidos, los resultados demuestran que la mayoría de las prácticas pedagógicas de los docentes no guardan relación con lo que piensan sobre E.I., y que, además, conciben el concepto inclusiva, solo desde la discapacidad.

Palabras Clave: docentes, educación inclusiva, diversidad, concepciones, prácticas pedagógicas.

\footnotetext{
* Universidad Católica Luis Amigó, Medellín, Colombia. ORCID ID: https://orcid.org/0000-0002-0876-8909

** I.E. Santa María Goretti. Montería - Córdoba, Colombia. ORCID ID: https://orcid.org/0000-0001-8795-0903
} 
Benítez, N. S., \& Pacheco, P. (2021). Concepciones sobre Educación Inclusiva y su relación con la práctica pedagógica de los docentes: un estudio de caso. Revista Convergencia Educativa, (9), julio, 16-29. DOI: http://doi.org/10.29035/rce.9.16

\begin{abstract}
Inclusive Education (IE) focuses on the attention to diversity, to the extent that, through administrative and pedagogical processes, it seeks to recognise and enhance the strengths of all students in vulnerable situations, taking into account their different styles, rhythms and particularities in learning. From this perspective, the present research developed in a Educational Institution in the city of Monteria-Cordoba. The study aimed at determining the relationships that exist between the conceptions that teachers of this institution have about inclusive education (IE) and the relationships that exist between these with the pedagogical practice that they develop in their classrooms. In order to achieve this goal, the methodology used was based on a qualitative approach, with a phenomenological design, which allowed the authors to highlight the experiences of the teachers and to rescue the immersed voices that show whether or not there is a relationship between what they say and what they do in terms of the implementation of IE. Three techniques were used to collect the information: a contextual reading, a semi-structured interview with 8 secondary school teachers from grades 6 to 11 and three different class sessions were observed by each teacher, recording what was observed through an observation protocol. The results obtained highlight that most of the pedagogical practices of teachers are not related to what they think about IE and that, in addition, they conceive the term inclusive only from the point of disability.
\end{abstract}

Key words: Teachers, Inclusive Education, diversity, conceptions, pedagogical practices.

\title{
1. PROBLEMA DE LA INVESTIGACIÓN
}

Esta investigación se centra en el ámbito educativo fijándose directamente en la práctica pedagógica, porque es en ella donde se evidencia el desarrollo de los procesos de E.I, los cuales se caracterizan por el reconocimiento de la diversidad desde varios aspectos. Éstos atañen a las particularidades de los estudiantes, evidenciadas en sus características de aprendizaje, identidades culturales y personales, condiciones de discapacidad, entre otras contextuales, como el nivel socioeconómico, situaciones relacionadas con el conflicto, y otras, que particularizan la vida del ser humano en sus diferentes dimensiones.

Desde esta perspectiva, cobra relevancia la investigación: Concepciones sobre Educación Inclusiva y su relación con la práctica pedagógica de los docentes de la Institución Educativa Santa María Goretti, ya que se centra en una mirada de la diversidad y no solo en uno de sus aspectos, como lo es la discapacidad, pues con ello, se estaría dejando de lado la participación del estudiantado que, por situaciones y condiciones diferentes a la mencionada, experimentan barreras para aprender, convivir o participar en los escenarios educativos.

En esta medida, se estaría fortaleciendo la práctica pedagógica haciéndola verdaderamente incluyente desde los espacios educativos mejorando y avanzando en la construcción de ambientes de bienestar para los estudiantes que impacten a la comunidad educativa en general. 
Benítez, N. S., \& Pacheco, P. (2021). Concepciones sobre Educación Inclusiva y su relación con la práctica pedagógica de los docentes: un estudio de caso. Revista Convergencia Educativa, (9), julio, 16-29. DOI: http://doi.org/10.29035/rce.9.16

Dentro de este orden de ideas, si no existe una práctica pedagógica incluyente se agudizan la discriminación, desigualdad, exclusión, deserción y mortalidad académica, dejando de lado el reconocimiento del otro desde sus particularidades, intereses, necesidades y derechos, reto fundamental del maestro en los procesos de E.I, tal como lo expresa Blanco (2008) al definir entre las tareas que este enfoque busca:

\footnotetext{
Identificar y minimizar las barreras que enfrentan los estudiantes para acceder y permanecer en la escuela, participar y aprender. Estas barreras surgen de la interacción entre el alumno y los distintos contextos: las personas, políticas, instituciones, culturas y las circunstancias sociales y económicas que afectan sus vidas.

En este sentido, las acciones han de estar dirigidas principalmente a eliminar las barreras físicas, personales e institucionales que limitan las oportunidades de aprendizaje y el pleno acceso y participación de todos en las actividades educativas (p.9).
}

\section{JUSTIFICACIÓN}

Dentro del ámbito educativo, existen referentes claves para el reconocimiento de un proceso de Educación Inclusiva tales como: currículo flexible, políticas institucionales en favor del reconocimiento a la diversidad, etc.; todos son importantes, pero conviene resaltar, en específico, las actitudes, atención, empeño y compañía, éstas pueden lograr que, dentro de una institución educativa y propiamente en un aula de clases, cada estudiante sienta que es visto desde lo que es, piensa y puede conseguir hacer.

Por lo anterior, un educador debe asumir su rol para atender y responder a la diversidad de sus estudiantes, no solo desde la intención sino también reformando su propia práctica pedagógica, en la medida de cada necesidad y lo que ésta exige, reconociendo que este proceso se logra llevar a cabo a través de un trabajo cooperativo entre los docentes; de acuerdo a Blanco (2008), quien manifiesta que:

\footnotetext{
Atender e integrar la diversidad en las aulas es una tarea compleja que requiere aumentar las motivaciones y competencias de los docentes, transformar la formación de los docentes y crear condiciones adecuadas de trabajo. Se necesita un docente que se atreva a asumir riesgos y esté abierto al cambio, que busque nuevas formas de enseñanza y reflexione de forma crítica sobre su práctica para transformarla, que valore las diferencias como un elemento de enriquecimiento profesional y que sea capaz de trabajar en colaboración con otros docentes, profesionales y familias (p.11).
}

Bajo estas condiciones, estamos frente a una nueva visión formativa y de desempeño respecto al cuerpo docente, que lo conduce a trabajar desde una reflexión constante sobre su práctica, de manera que combine la sensibilidad frente a las condiciones y situaciones de sus estudiantes, para emplearlas a su favor durante el proceso de enseñanza, para, en consecuencia, presentarles una gama de posibilidades didácticas que permitan pasar de su nivel de aprendiz básico a un nivel más avanzado. 
Benítez, N. S., \& Pacheco, P. (2021). Concepciones sobre Educación Inclusiva y su relación con la práctica pedagógica de los docentes: un estudio de caso. Revista Convergencia Educativa, (9), julio, 16-29. DOI: http://doi.org/10.29035/rce.9.16

\section{METODOLOGÍA}

En esta investigación, se empleó un enfoque cualitativo, el cual permite ver cada situación de forma natural, es decir, desde las propias percepciones que las personas otorgan, lo cual permite interactuar con ellas desde las concepciones que tienen sobre Educación Inclusiva, analizando a su vez sus prácticas pedagógicas, lo que hace directamente un trabajo con la comunidad desde las aulas de clase, haciendo una interpretación de las narraciones descritas en la entrevista y lo observado en la realidad.

Para el desarrollo de la investigación, se utilizó el diseño fenomenológico el cual es comprendido como el proceso donde el eje central es el ser humano y sus vivencias, quienes determinan en él su proceder basado en el concepto que tenga sobre lo que está viviendo. De esta manera, Fuster (2019), manifiesta que:

...por su naturaleza se enfoca en las vivencias y destaca el sentido que envuelve lo cotidiano, el significado del ser humano, es decir, la experiencia que somos. La fenomenología es sensible a la problemática desatada en torno al mundo de la vida (p.8).

En coherencia con dicho diseño, se utilizaron como técnicas de recolección de información: lectura de contexto, entrevista semi-estructurada y observación, las cuales permitieron estructurar la organización de la investigación en cuanto a las etapas a seguir según los objetivos propuestos.

Para la selección de la muestra se tuvieron en cuenta criterios como: conocer la investigación, participar en la entrevista semi-estructurada, permitir la observación realizada en el aula, tener dos o más años de experiencia en el sector educativo y al menos un año de experiencia en la Institución Santa María Goretti y ser docente por lo menos de un grado de $6^{\circ}$ a $11^{\circ}$.

Con respecto a la población, está determinada por los docentes que pertenecen a la Institución Educativa Santa María Goretti de la ciudad de Montería-Córdoba; la muestra la conforman ocho docentes que, informados de la investigación, aceptan los demás criterios para ser parte de la muestra seleccionada, como su participación en la entrevista semi-estructurada, la cual consta de 13 preguntas abiertas para conocer las concepciones sobre E.I. y permitir la observación realizada en el aula, de acuerdo al protocolo de observación construido, con el fin de encontrar si existe relación entre lo que dicen y hacen.

\section{ANÁLISIS Y DISCUSIÓN}

$\mathrm{Al}$ iniciar este apartado, es preciso tener en cuenta los antecedentes y actividades previas a la adquisición de los resultados y el orden de las instancias de la investigación, es decir, primero hablar sobre la lectura de contexto, luego de las entrevistas y finalmente de las observaciones de las intervenciones pedagógicas de los docentes. 
Benítez, N. S., \& Pacheco, P. (2021). Concepciones sobre Educación Inclusiva y su relación con la práctica pedagógica de los docentes: un estudio de caso. Revista Convergencia Educativa, (9), julio, 16-29. DOI: http://doi.org/10.29035/rce.9.16

\section{a. Lectura de contexto:}

En esta primera parte, se caracterizó el contexto social y educativo de los estudiantes, a nivel de la familia, barrio, comunidad y aula de clases, con el fin de conocer cada uno de estos aspectos y analizar cómo influyen en el proceso de formación de los educandos.

\section{b. Entrevista:}

De acuerdo al análisis de las respuestas de la entrevista se tiene como resultado específico que en la entrevista manifiestan concebir la Educación Inclusiva (E.I.) solo desde la discapacidad.

\section{c. Observación:}

Con relación a la entrevista, de igual forma se evidencia que los docentes no identifican ritmos y estilos de aprendizaje de sus estudiantes, en la medida en que no se percatan por qué muchos de sus educandos no están participando en clases, pues puede que esto implique diversas señales y no sean advertidas por el docente, al tiempo que demuestran más interés en el desarrollo de las habilidades intelectuales.

Por otra parte, los educadores expresan básicamente que, al detectar las limitaciones y dificultades a nivel general, hacen los ajustes pertinentes a las clases, sin embargo, son muy pocos los docentes que llevan esto a la práctica.

Los resultados mencionados solo demuestran que los docentes de la Institución Santa María Goretti, pese a que conciben la Educación Inclusiva desde la discapacidad nada más, la mayoría se hace también consciente de que los ajustes deben hacerse, pero muy pocos están desarrollando esta parte en sus aulas.

Lo anterior, no está dado bien sea porque conciben el proceso como un programa impuesto por el Ministerio de Educación Nacional (MEN) según lo manifestado por ellos mismos, o bien porque para los docentes concentrarse en las limitaciones y dificultades de los estudiantes, implica retrasar el proceso académico y se pone en juego la calidad de la institución educativa.

Sin embargo, este análisis debe ir precisando aspectos que para los educadores deben quedar claros, esto significa que los resultados de esta investigación muestran directamente que no existe una relación entre lo que conciben los docentes sobre Educación Inclusiva y lo que hacen en su práctica de aula.

Ahora bien, esto en gran parte, es ocasionado porque la mayoría de ellos centra la Educación Inclusiva en un solo aspecto: la discapacidad, lo cual no les está permitiendo ver y asumir la E.I. como un proceso donde día a día se reconoce, identifica y atiende la diversidad como una parte que enriquece la práctica educativa. 
Benítez, N. S., \& Pacheco, P. (2021). Concepciones sobre Educación Inclusiva y su relación con la práctica pedagógica de los docentes: un estudio de caso. Revista Convergencia Educativa, (9), julio, 16-29. DOI: http://doi.org/10.29035/rce.9.16

Con relación a lo anterior, Arnaiz (2016) expresa:

Hoy en día, la creciente diversidad de alumnos en nuestro sistema educativo es un importante tema de debate y preocupación. Entre las diferencias se encuentran la lengua, la cultura, la religión, el sexo, la discapacidad [...] y muchas más que denotan la multiculturalidad existente en la escuela. Pero, frente a esta realidad, a menudo encontramos que la diversidad es entendida como un problema, más que como una maravillosa oportunidad de aprender sobre la variedad de vida de otras personas, y también sobre lo que significa ser humano (p.14).

Lo citado anteriormente, subraya que la discapacidad es solo una de las características que puede estar presente en el sujeto y que, por lo tanto, hace parte de su diversidad, la misma que es una realidad en el aula todos los días y debe ser vista como aprender de los demás, reconociendo en ellos la otredad como punto clave para apoyarse entre todos, incluidos en un mismo proceso, pero atendiendo las necesidades de cada uno.

Ahora bien, en lo que tiene que ver con las prácticas inclusivas realizadas en el aula, algunos de los docentes entrevistados expresaron que en sus clases desarrollan estrategias con directrices establecidas, es decir, van siempre ligadas a lo que la Institución propone a nivel general y como docentes no fortalecen el desarrollo de la autonomía en el proceso educativo, estrategias como actividades en clases, talleres de nivelación, así como conocer particularidades de los estudiantes que hacen parte de la dirección de grupo del docente.

Conforme a lo expresado por algunos educadores en cuanto a no sentirse autónomos en lo que puedan desarrollar en el aula, surge el hecho de pensar que se encuentran en una dicotomía respecto a qué es lo que deben hacer según algunas pautas institucionales comunes, frente a qué quieren hacer ellos como educadores autónomos en sus sesiones de clases.

Para los docentes es difícil muchas veces reaccionar frente a lo que se debe cumplir y lo que, según ellos, puede desarrollarse también en el salón de clases para alcanzar los objetivos propuestos y atender necesidades de los educandos, lo que lleva, según ellos, a cohibirse en ocasiones de llevar a cabo algunos procesos en pro de lo mencionado.

En este aspecto, Duk \& Murillo (2016) sostienen:

El dilema fundamental que se mueve alrededor de los sistemas generales de educación, dice relación con cómo lograr el difícil equilibrio ente lo común y lo diverso. Es decir, el dilema de cómo ofrecer una educación pertinente, ajustada a las diferencias socioculturales e individuales de los y las estudiantes y, al mismo tiempo, hacerlo en el marco de estructuras comunes e inclusivas (sistema, escuela y aula), que garanticen condiciones de igualdad y favorezcan la convivencia en la diversidad, el sentido de pertenencia y la cohesión social (p.12). 
Benítez, N. S., \& Pacheco, P. (2021). Concepciones sobre Educación Inclusiva y su relación con la práctica pedagógica de los docentes: un estudio de caso. Revista Convergencia Educativa, (9), julio, 16-29. DOI: http://doi.org/10.29035/rce.9.16

Lo expresado por los autores, precisa lo dicho sobre el dilema de los docentes sobre permitirse realizar ajustes a las clases, en la medida de las necesidades de los estudiantes y tener que cumplir con parámetros institucionales que deben marcar la calidad educativa.

Por otro lado, la mayoría de los docentes asumieron que en la práctica de aula hacen más énfasis en las características intelectuales de los estudiantes que en otras, puesto que la evaluación se ve como un producto final, se dejan compromisos al estudiante sobre una consulta del tema que sigue, pero solo con la intención de que tenga argumentos con qué participar en la clase, de igual forma al docente le interesa la profundidad con que el educando ha visto el tema, solo por saber qué estudiantes son sobresalientes en el desarrollo de las temáticas.

Asimismo, las oportunidades dadas al educando se ofrecen solo si él las pide y son dadas poniendo límites de tiempo, debido a que hay que cumplir contenidos y avanzar, además en esta parte intelectual, según los docentes, está en juego la calidad educativa de la institución y es algo importante para el avance del establecimiento.

Sin embargo, no es lo único que se debe tener en cuenta en el proceso formativo, pues el sujeto no se resume solo en la parte cognoscente, hacen parte otros desarrollos y otras esferas como la emocional, física, axiológica, entre otras que lo conforman como ser humano.

De acuerdo a lo anterior, es claro que en la institución es relevante el tema de la calidad educativa, pero no se ha tenido en cuenta que al poner en práctica la Educación Inclusiva a nivel institucional, e incluso, no solo en el aula, se estará logrando la calidad educativa que debe caracterizar a cada institución, pues de esta manera si hay equidad, existirá calidad, por tanto, debe reestructurarse el pensamiento sobre lo que debe considerarse la calidad educativa.

Dicho en una forma más específica por Marchesi (2014):

Tal vez sea posible una propuesta algo más completa, que incluya también un referente a la equidad educativa, indisociable de la calidad para ello, debería de tenerse en cuenta [...] cuidado del contexto sociocultural de los alumnos [...] competencias y sensibilidad de los profesores para enseñar bien a todos sus alumnos y adaptarse a sus posibilidades; orientación equilibrada hacia los objetivos de la educación: conocer, hacer, convivir y ser; atención a los alumnos con dificultades de aprendizaje para reducir lo más posible las diferencias entre ellos (p.43).

La cita demuestra que existen ciertas especificaciones que todo ente educativo debe concebir como parte fundamental de la calidad educativa, puesto que son aspectos inherentes a la práctica de aula y que todo docente e institución en general debe asumir con propiedad. 
Benítez, N. S., \& Pacheco, P. (2021). Concepciones sobre Educación Inclusiva y su relación con la práctica pedagógica de los docentes: un estudio de caso. Revista Convergencia Educativa, (9), julio, 16-29. DOI: http://doi.org/10.29035/rce.9.16

En lo que se refiere a la observación de las clases, en cuanto a la organización del salón, la mayoría de los docentes no tienen en cuenta su disposición según las diversas necesidades de los estudiantes, es más, pasan por alto condiciones particulares y determinantes, por ejemplo, si son zurdos, con hipoacusia u obesidad mórbida, entre otras, interviniendo este orden solo cuando los afectados se acercan a manifestar su dificultad; en efecto, al momento de distribuir la audiencia, éstos jerarquizan la estatura, para mantener las filas según este criterio, además de velar por un aula limpia, y, en definitiva, los cambios de puestos se imponen por actos de indisciplina.

En esta medida, organizan de manera diferente al grupo en cuanto al espacio, ya sea en mesa redonda o semicírculo solo si dentro de las actividades a desarrollar tienen una exposición o también si se proyectará un video en el salón.

La organización del aula es un aspecto que los docentes deben tener presente, no solo para que se vea limpia y adecuada para desarrollar las temáticas, sino más que todo pensando y atendiendo las necesidades de los estudiantes, con el fin que se sientan cómodos en un ambiente donde vean que son aceptados y reconocidos con sus características, dentro de un numeroso grupo de trabajo y convivencia.

De acuerdo con esto, Crisol (2015) plantea:

Las aulas representan el lugar por excelencia de acogida del alumnado en el centro educativo. Además de considerarse el lugar donde suelen pasar la mayor parte del tiempo; de ahí que jueguen un papel decisivo en el proceso de inclusión puesto que se constituyen en comunidades que dan la bienvenida a la diversidad, honrando y respetando las diferencias (p.221).

El autor precisa que es en el aula, donde, al convivir unos con otros en el transcurrir de actividades, se logra que se valore la diversidad, en tanto que es allí donde se deben reconocer las distintas características de los estudiantes, logrando que entre todos se fortalezca el aprendizaje, independientemente de cualquier temática a desarrollar.

Respecto a lo observado acerca de promover el respeto por la diversidad en el aula, es sorprendente ver que los docentes solo lo hacen si se da una situación de conflicto en el salón, porque los educadores deben ser los primeros en reconocer y, más aún, poner en práctica el hecho de promover día a día el respeto en las aulas, porque no es válido esperar a que se dé el conflicto para empezar a hablar de este valor.

Por esto es relevante, antes de implementar estrategias de trabajo en clases en el aula, priorizar la comunicación entre estudiantes y docentes, donde se establezca el diálogo en la resolución de conflictos como primera herramienta y, más aún, que, a través de él, en cada sesión se fortalezca el respeto frente a la particularidad de cada educando y por tanto se valore. 
Benítez, N. S., \& Pacheco, P. (2021). Concepciones sobre Educación Inclusiva y su relación con la práctica pedagógica de los docentes: un estudio de caso. Revista Convergencia Educativa, (9), julio, 16-29. DOI: http://doi.org/10.29035/rce.9.16

De esta manera es expuesto por Cárdenas \& Aguilar (2015):

El diálogo es una herramienta para la diversidad, sirve como puente entre cada uno de los actores educativos, acercándolos para traducir las otras formas de pensar y vivir, promoviendo la gestión de conflictos por medios pacíficos y el establecimiento de una convivencia construida por las diferencias (p.182).

Lo que se cita en el párrafo anterior, es lo que se debe tener en cuenta, en la estructura de toda sesión de clase: inicio, desarrollo y finalización, pues del diálogo y confianza en el aula dependerá que se valore la presencia del otro, en la medida en que se logre el reconocimiento de lo que un compañero puede aportar a los demás y viceversa.

En la parte de la evaluación, los docentes varían en tipos y formas, pues la mayoría emplea diversas maneras de evaluar: auto, hetero y coevaluación, así como los talleres, exposiciones, sin embargo, por lo general priman las evaluaciones y talleres escritos, así como la participación en clases.

En lo que compete a las actividades de recuperación, algunos docentes conceden a los estudiantes distintas oportunidades de recuperación durante el proceso, no al final; otros educadores, muy apegados a la norma institucional, realizan las nivelaciones en un tiempo en específico y bajo parámetros estrictos.

La evaluación antes que un producto debe ser un proceso, donde el estudiante reconozca y afiance sus potencialidades, las mismas que harán de él un ser con características propias que lo identificarán frente a los demás.

Lo anterior quiere decir una vez más que el docente es el encargado principal de que esto se logre, por esta razón debe variar consecutivamente los tipos y formas de evaluación, con el fin de tener presente la diversidad en el aula, mostrando que es posible atender el potencial que tiene cada uno de los estudiantes desde sus propias cualidades.

Así lo asume Blanco (2017):

Una verdadera educación inclusiva se ve reflejada en un aspecto esencial del proceso enseñanzaaprendizaje como lo es la evaluación. Solo se logrará una educación inclusiva cuando se practique un sistema de evaluación que respete y valore las diferencias individuales (p.59).

En este sentido, es necesario mencionar la forma como debe concebirse un aula incluyente, dado que no se trata de ofrecer un instructivo a seguir, simplemente es reorganizar un aula donde quepan todos, pero más que en un espacio físico en un proceso continuo y equitativo. En esta medida, el docente solo debe llegar al salón de clases reconociendo la variedad de características que lo recibirá en el aula, las cuales aportarán un poco cada día a él como ser humano. 
Benítez, N. S., \& Pacheco, P. (2021). Concepciones sobre Educación Inclusiva y su relación con la práctica pedagógica de los docentes: un estudio de caso. Revista Convergencia Educativa, (9), julio, 16-29. DOI: http://doi.org/10.29035/rce.9.16

Por esta razón, algunos autores plantean muy bien lo que tiene que ver con la concepción que debe tenerse de Institución-aula desde la Educación Inclusiva. En este sentido, Andújar \& Rosoli (2014) expresan:

En las escuelas donde se atiende la diversidad, el aula es un espacio dinámico donde cada día o semana el ambiente y la disposición del mobiliario pueden adaptarse de acuerdo a las necesidades del grupo y de las competencias a desarrollar. Este dinamismo favorece el uso constante de todos los materiales y recursos con los que se cuenta, tanto en el aula como en la escuela.

Con esta filosofía de trabajo los estudiantes construyen conocimiento entre todos, los más avanzados apoyan a los que aún no han logrado ciertas competencias, el conocimiento circula y se valoran y reconocen los esfuerzos y logros de todos (p.55).

Cuando se habla de Educación Inclusiva, esta cita debe ser tenida en cuenta por los docentes, pues este proceso permite, además, visualizar nuevos proyectos en pro del bienestar de todos los educandos, pues cada logro significa un recurso para las nuevas generaciones profesionales en la docencia, para prevenir divisiones en la sala de clases, y, en consecuencia, segmentar la educación, como se ha visto hasta ahora.

De hecho, parte de lo encontrado en las entrevistas recoge lo que debe ir dejándose atrás frente a lo que es exactamente la Educación Inclusiva y para esto se debe partir de aceptar que ha existido un pensamiento dividido sobre este tema.

Wehmeyer (2009) sustenta que se dio un primer momento donde los estudiantes segregados por alguna limitación pasaban a ser incluidos en las clases. Otro donde las instituciones buscaban estrategias de apoyo para estudiantes con discapacidad, entonces es hora de pasar a ese tercer momento o tercera generación, es decir, donde se cambia de enfoque mirando al estudiante en sí y no la adaptación del lugar donde se encuentra.

Para complementar lo anterior, Ocampo (2012) plantea:

Abordar institucionalmente, un proceso de inclusión socioeducativa es asumir una transformación ideológica de la escuela, respecto de los procesos prácticos y de las visiones organizativas, que sustentan y representan la "diversidad" al interior de la escuela y de su comunidad.

No obstante, ninguna transformación logrará impactar, sustantivamente en sus estructuras, si es que, no se reflexiona, sobre ¿qué es la escuela hoy? y, sobre la necesidad de hacer explícito en su discurso, al sujeto de la educación (p.135).

El autor destaca el cambio de concepción sobre la atención de la diversidad en el aula centrada en la Educación Inclusiva, pues se trata de pensar en el estudiante y en lo que se puede lograr desarrollar en él, 
Benítez, N. S., \& Pacheco, P. (2021). Concepciones sobre Educación Inclusiva y su relación con la práctica pedagógica de los docentes: un estudio de caso. Revista Convergencia Educativa, (9), julio, 16-29. DOI: http://doi.org/10.29035/rce.9.16

en la medida en que lo demuestre desde su particularidad, por sobre la pretensión de que encaje en lo que ya está estipulado y, paralelamente, a lo que debe ajustarse.

Para sintetizar, el docente es el principal actor del proceso de Educación Inclusiva, puesto que por medio de él los estudiantes aprenderán o no a reconocer, identificar y valorar la diversidad. En la medida en que el educador impulse día a día el respeto por el otro, todos los educandos multiplicarán cada acto que valore las características del que tienen al lado o al frente, comprendiéndolo desde lo que es y no desde lo que otras personas quieren que sea.

\section{RESULTADOS}

Conforme a los resultados de la entrevista, es relevante el hecho de haber encontrado en lo dicho por la mayoría de los docentes, que para ellos la Educación Inclusiva es sinónimo de discapacidad; explicando que, para ellos, la E.I., significa netamente la atención a dicha población, esto implica, por tanto, tener la disposición de aprender más sobre los estudiantes que se encuentren en esta condición, pues esto conlleva a acercarse más al estudiante y conocer un poco más sobre él.

En esta medida, la mayoría de los docentes entrevistados, al tener estas concepciones, expresan también la necesidad de hacer sus ajustes a las clases, como emplear el trabajo colaborativo o implementar actividades diferentes, para, de tal forma, facilitar al estudiante con discapacidad, la oportunidad de ser partícipe de cada sesión y actividad.

Adicionalmente, se encuentra que los docentes conciben la E.I como un programa del Ministerio de Educación y no como una alternativa educativa para dar respuesta a la diversidad inmersa en el aula y, por tanto, se instaura en las prácticas docentes, así como en las políticas y cultura de las instituciones educativas.

Complementariamente, en la observación en el aula, se puede resaltar que se tuvo la oportunidad de estar presente en tres sesiones de clase, de cada uno de los docentes, lo que llevó a fijarse muy bien según el protocolo de observación, si están siendo o no incluyentes en sus prácticas de aula.

Lo anterior dejó ver que son muy pocos los docentes incluyentes en su aula de clases, puesto que la mayoría está más pendiente de cumplir con los plazos indicados para las temáticas y horarios, de avanzar especialmente con los estudiantes más participativos en clases y, sobre todo, de desarrollar las habilidades intelectuales en los educandos.

\section{CONCLUSIONES}

En el desarrollo de la investigación, se aplicaron varios instrumentos que permitieron tener los resultados frente a, si existe o no, relación entre lo que conciben los docentes sobre Educación Inclusiva y lo que hacen en su práctica de aula. 
Benítez, N. S., \& Pacheco, P. (2021). Concepciones sobre Educación Inclusiva y su relación con la práctica pedagógica de los docentes: un estudio de caso. Revista Convergencia Educativa, (9), julio, 16-29. DOI: http://doi.org/10.29035/rce.9.16

Conforme a lo encontrado, se determinó que no existe esa relación pues la mayoría de los docentes concibe la E.I. solo desde la discapacidad y, a su vez, expresan hacer ajustes en su práctica de aula, lo cual no se evidenció en la observación.

En esta medida, cuando los docentes conciben la Educación Inclusiva únicamente desde la discapacidad, sin tener en cuenta que ella es solo una de las características que la sustentan, empiezan a expresar lo que según ellos implica realizar en el aula, como tener una disposición por parte de toda la institución al incluir estudiantes con algunas limitaciones, tratarlos diferente, en el sentido de realizar actividades diferentes para ellos e incluso lo que implica más esfuerzo para ellos como docentes: asumir compromisos en el aula, sin apoyo de especialistas.

Todo lo anterior lleva a que los docentes no identifiquen otras características de sus estudiantes como son los ritmos y estilos de aprendizaje o que tengan en cuenta la organización del aula y presentación de la información teniendo en cuenta las dificultades de los estudiantes, como no ver o escuchar muy bien de lejos, ofrecerles distintas formas de iniciar una clase con el fin de darle oportunidad a cada uno de participar desde su comodidad.

Ahora bien, estas conclusiones son producto del análisis de la entrevista a cada docente y lo observado en el aula. Con estos antecedentes, es posible determinar que los educadores entrevistados se manifiestan acerca de tener en cuenta aspectos relacionados a la evaluación en sus clases, como la aplicación de diversos tipos y formas, organizar el salón de clases según las necesidades de los estudiantes y motivar a los menos participativos; sin embargo, en la práctica pedagógica muchos de ellos desarrollan las clases sin tener en cuenta nada de lo anterior.

No obstante, dentro de lo expresado en la entrevista, algunos docentes evidencian que pensar en E.I. es vivir la realidad en el aula, pues requiere exponer el tema a los estudiantes procurando su comprensión y, en todo momento, reconocer que cada uno tiene una forma particular de aprender, además de conducir el reconocimiento entre pares, identificando las cualidades de cada uno, que sumadas y en conjunto permitirán alcanzar los objetivos propuestos, valorando el aporte de cada uno.

De ahí que, al sintetizar todo lo que ha sido el proceso investigativo, no se debe olvidar hacer mención específicamente de algunas de las metas que están proyectadas para cumplirse en 2021 respecto a la Educación Inclusiva, como lo es organizar un currículo flexible desde la atención a la diversidad, es decir donde sea permitido el pleno desarrollo de distintos tipos de capacidades o fortalezas de los estudiantes, así como centrarse en la formación docente.

Con relación a la formación docente, el objetivo de las capacitaciones es lograr que los educadores sean conscientes de que la Educación Inclusiva no consiste en prepararse para desarrollar una clase diferente para cada estudiante en un salón, sino simplemente identificar y atender en el aula la particularidad de cada 
Benítez, N. S., \& Pacheco, P. (2021). Concepciones sobre Educación Inclusiva y su relación con la práctica pedagógica de los docentes: un estudio de caso. Revista Convergencia Educativa, (9), julio, 16-29. DOI: http://doi.org/10.29035/rce.9.16

uno de ellos, sin hacer distinciones, solo respetándola y valorándola como lo que enriquece la práctica pedagógica.

En conclusión, es notable que a la fecha las metas anteriormente mencionadas, no han tenido los avances esperados, lo cual se ha logrado evidenciar en los resultados de la presente investigación, pues aún el currículo sigue sin ser flexible respecto a las características de los estudiantes y los docentes continúan viendo la Educación Inclusiva como caso aparte y no como un proceso que es totalmente inherente a la Educación cotidiana, desarrollada día a día en el aula.

\section{REFERENCIAS BIBLIOGRÁFICAS}

Andújar, C., \& Rosoli, A. (2014). Enseñar y aprender en la diversidad: el desarrollo de centros y aulas inclusivas. En A. Marchesi, R. Blanco, \& L. Hernández (Coord), Avances y desafíos de la educación inclusiva en Iberoamérica, (pp. 47-59). Organización de Estados Iberoamericanos para la Educación, la Ciencia y la Cultura. https://www.oei.es/uploads/files/microsites/28/140/metasinclusiva.pdf

Arnaiz, P. (2016). Sobre la atención a la diversidad. Universidad de Murcia. https://www.slideshare.net/mizhellagalleta/sobre-la-atencin-a-la-diversidad

Blanco, R. (2008). Marco Conceptual sobre educación inclusiva. Cuadragésima octava Conferencia Internacional de Educación Ginebra, Suiza. http://www.ibe.unesco.org/fileadmin/user_upload/Policy_Dialogue/48th_ICE/CONFINTED_48_I nf_2_Spanish.pdf

Blanco, R. (2017). La evaluación al servicio del aprendizaje de todos... una evaluación inclusiva. Boletín Virtual, 6(8), 57-62. https://dialnet.unirioja.es/servlet/articulo?codigo=6224325

Cárdenas, B., \& Aguilar, M. (2015). Respeto a la diversidad para prevenir la discriminación en las escuelas. Ra-Ximhai, 11(1), 169-186. https://www.redalyc.org/pdf/461/46139401009.pdf

Crisol, E. (2015). Hacia un aula inclusiva. Condiciones didácticas y organizativas. Dialógica: Revista multidisciplinaria, 12(2), 217-258. https://dialnet.unirioja.es/servlet/articulo?codigo=6224439

Duk, C., \& Murillo, J. (2016). La Inclusión como Dilema. Revista Latinoamericana de Educación Inclusiva, 10(1), 11-14. http://dx.doi.org/10.4067/S0718-73782016000100001 
Benítez, N. S., \& Pacheco, P. (2021). Concepciones sobre Educación Inclusiva y su relación con la práctica pedagógica de los docentes: un estudio de caso. Revista Convergencia Educativa, (9), julio, 16-29. DOI: http://doi.org/10.29035/rce.9.16

Fuster, D. (2019). Investigación cualitativa: Método fenomenológico hermenéutico. Revista Propósitos y Representaciones, 7(1), 201-229. http://dx.doi.org/10.20511/pyr2019.v7n1.267

Marchesi, A. (2014). Retos y dilemas de la inclusión educativa iniciales. En A. Marchesi, R. Blanco, \& L. Hernández. (Coord), Avances y desafíos de la educación inclusiva en Iberoamérica (pp. 37-45). https://www.oei.es/uploads/files/microsites/28/140/metas-inclusiva.pdf

Ocampo, A. (2012). Mejorar la escuela inclusiva: Un desafío de todos. Revista Educación Inclusiva, 5(3), 133141. https://revistaeducacioninclusiva.es/index.php/REI/article/view/248/242

Wehmeyer, M. (2009). Autodeterminación y la Tercera Generación de prácticas de inclusión. Revista de Educación, (349), 45-67. https://dialnet.unirioja.es/servlet/articulo?codigo=2995005

\section{Datos de correspondencia}

Mg. Nilsa Shirley Benítez

Magíster en Educación

Universidad Católica Luis Amigó

Medellín, Colombia.

Dirección postal: Calle 89b\# 72-128, Medellín, Colombia.

Código postal 050040

ORCID ID: https://orcid.org/0000-0002-0876-8909

Email: nilsashirley@gmail.com 